\title{
Impact of Heterogeneity on Opinion Dynamics: Heterogeneous Interaction Model
}

\author{
Xi Chen, Zhan Wu, Hongwei Wang, and Wei Li \\ School of Automation, Huazhong University of Science and Technology, Key Laboratory of Ministry of Education for \\ Image Processing and Intelligent Control, Wuhan 430074, China
}

Correspondence should be addressed to Wei Li; liwei0828@mail.hust.edu.cn

Received 19 December 2016; Accepted 22 March 2017; Published 24 April 2017

Academic Editor: Alicia Cordero

Copyright (C) 2017 Xi Chen et al. This is an open access article distributed under the Creative Commons Attribution License, which permits unrestricted use, distribution, and reproduction in any medium, provided the original work is properly cited.

Considering the impact that physical distance and other properties have on the change of opinions, this paper introduces an intension model of the Hegselmann-Krause (KH) model-heterogeneous interaction (HI) model. Based on the classical KH model, $\mathrm{HI}$ model designs new interaction rules and the interactive radius considering the impact of heterogeneous attributes, such as physical distance, individual conformity, and authority. The experiment results show that the opinion evolution of the HI model will be similar to the classic $\mathrm{KH}$ model when the interactive radius is above the particular threshold value $(\sigma>600)$. Unlike the $\mathrm{KH}$ model, which leads to the polarization phenomenon; most agents reach a consensus in HI model when the confidence radius equals 0.2 , and the interactive radius remains within regulatory limits $(150<\sigma<520)$. The conclusions show that interactive radius affects public opinion evolution. HI model can explain more conscious opinion evolution in real life and has significance that effectively guides public opinion.

\section{Introduction}

As a social phenomenon in the social system, public opinion has attracted considerable interest from researchers in different scientific fields. A news article may spread globally in just a few seconds and further becomes a macroscopic network for public opinion [1]. Such rapid transmission can adversely affect people's normal and orderly lives, a state which may have considerable effect on the order of our society. If the propagation law of public opinion in communications media and new media is allowed to play out, it will result in negative consequences for social stability in certain locales and situations. Public opinion evolution is a highly complex process [2] and it is influenced by individual and environmental factors with dynamics [3]. The evolutionary model of public opinion is becoming a focal point for research in the study of public opinion.

At present, the most frequently used opinion dynamics models include the Deffuant model [4], Hegselmann-Krause model [5], and Sznajd model [6]. The KH model is the typical model. At each discrete time, every individual sets the average value of the neighbor's opinion, which is less than a confidence threshold, as the next moment individual's opinion in the $\mathrm{KH}$ model. With the change in size of the confidence threshold, the overall evolution of public opinion is divided into discrete, polarization, and consensus [7, 8], which is similar to real life situations. In real life, however, the update of an individual's opinion will be affected by a variety of aspects, such as psychological factors and social influence. The evolutionary process will be more complex.

In a recent study, considering the different environments and conditions in the evolution process, many improved $\mathrm{KH}$ models were put forward. When public opinion evolution occurred on the Internet, considering that the individual is unable to obtain all individuals' opinions, Chen et al. [9] proposed a high-effect priority bounded confidence model based on confidence threshold and social influence and it contains a dual mechanism. In real life, all individuals are not the same, and their confidence thresholds are different. Lorenz [10] proposed an improved $\mathrm{KH}$ model, wherein the confidence threshold is heterogeneous, and divided the agents into open-minded and closed-minded agents. Considering the influence of authority control on the public opinion, Chen et al. [11] proposed a network model of 
pinning control. Considering the opinion exchange between individuals is not decided by unilateral decision, the model considers the interplay between individual ability factors; Liang et al. [12] put forward an opinion dynamics model based on heterogeneous confidence parameters and social power; but this model only considers the heterogeneity of individual confidence threshold, without fully considering individual heterogeneity. In 2013, Jalili [13] put forward an opinion evolution model based on the difference in individuals and imported social influence on a small number of individuals and then used this small group to guide attitude change. This model defines social influence that is based on the difference of the network topology and ignores the individual's heterogeneity on the role of social influence. Although the above models consider many factors during evolution, they still lack consideration of some aspects, such as individual heterogeneity and interactive radius.

Previous studies have explained the public opinion phenomenon in a real society but rarely consider the impact of spatial constraints on opinion exchange. They assume that individuals are homogenous and failed to consider different individual attributes. In fact, full contact of any person to all the other individuals is impossible. As personal resources are finite, there is a limit on communication space, assumed as spatial constraints. Through long-term survey, psychologists found that with closer geographical location, individuals have more communication and interaction. Li and Zhang introduced an eyeshot mechanism similar to spatial constraints and investigated the effect of eyeshot on opinion dynamics. However, the model is set on square lattices, and individual property heterogeneity is not considered [14]. If all other conditions remain the same, adjacent people have more opportunity to meet with each other and know more about each other and, thus, are more likely to form a trust relationship. Individuals, who live in different localities, form social groups with different languages, cultural background, religious belief, and national tradition owing to the natural geological barriers. The same social groups of people have a higher recognition and trust level, and the communication between each other is more easily and more likely to have a similar attitude because of factors such as language and culture. Communication between individuals of two different social groups may produce the opposite results owing to background difference. In general, language, cultural background, religious beliefs, traditional factors, and so forth have the characteristics of geographical location convergence.

In this thesis, we introduce spatial constraints into modeling the evolution process of public opinion. Local communication is formed under the limitation of physical distance between two individuals. Combined with the researches in psychology and sociology, new factors including individual attributes, personality characteristics, and public authority are expressed quantitatively. Using the agent-based method, an evolutionary model of public opinion based on physical distance is developed. Through comparisons with two classical bounded confidence models, our results are in good agreement with the new features in the expansion of human communication and provide a comprehensive explanation for the evolution of public opinion in real social systems.

\section{Heterogeneous Interaction Model}

In this section, we construct an evolution model-Heterogeneous Interaction Model (HI). Firstly, we provide a detailed illustration about some related definitions, which play a vital role during the evolution process. Based on these preliminaries, we provide a description of some assumptions about the model. Subsequently, we present the algorithm of HI model.

2.1. Attributes of Individuals. In previous studies of opinion modeling, the heterogeneity of individuals has been unrepresented and individuals in social networks were assumed to be identical. It is evident that different social individuals manifest various social attributes and have different ways of forming their opinions. In our model, several important influence factors are abstracted and quantified to represent the heterogeneity among individuals.

(1) Character Trait. It can be concluded that opinion formation is greatly affected by character trait of a person. In the real world, the degree of belief in rumors depends on the individual itself. A conformity parameter is defined to measure the different degree of opinion variation. $\lambda_{i}$ is assigned to agent $i$ to denote the individual conformity [15] of agent $i$. Extremists are introduced on behalf of the type of people who refuse to change their attitude or wholly follow others in the evolution process. In general, extremists only take a small portion. Conformity parameter assignment is based on the principle of $80 / 20$. The Italian economist Pareto proposed the $80 / 20$ rule. He believes that for many events, the most important part, which plays a decisive role, is only approximately $20 \%$ and the remaining $80 \%$ is secondary and nonconclusive. After long-term exploration, many imbalance phenomena in life have been discovered that can be explained by the $80 / 20$ rule, which has been widely used in fields such as sociology and business management. In our model about $20 \%$ of people are considered as extremists and $80 \%$ of individuals are ordinary individuals. The values are taken into account according to the following criteria (Figure 1):

(a) $10 \%$ of individuals are the dogmatic people with $\lambda_{i}=$ 0.1 .

(b) $10 \%$ of individuals are the copycats with $\lambda_{i}=0.9$.

(c) $80 \%$ of individuals are the easy-going people with $\lambda_{i}=$ 0.5 .

(2) Geographic Position. Spatial constraints are quantified through the physical distance between two individuals. Hence, geographic position is introduced to compute the physical distance between two individuals. Geographical position determines the community a person belongs to. The physical distance between two individuals determines local communication with others in the system, which is a better way to describe information exchange in a so-called realistic society. The reference collection one can communicate with is decided by geographic position, which is the core condition for modeling opinion dynamics. We use two-dimensional coordinates to represent geographic positions of individuals. 


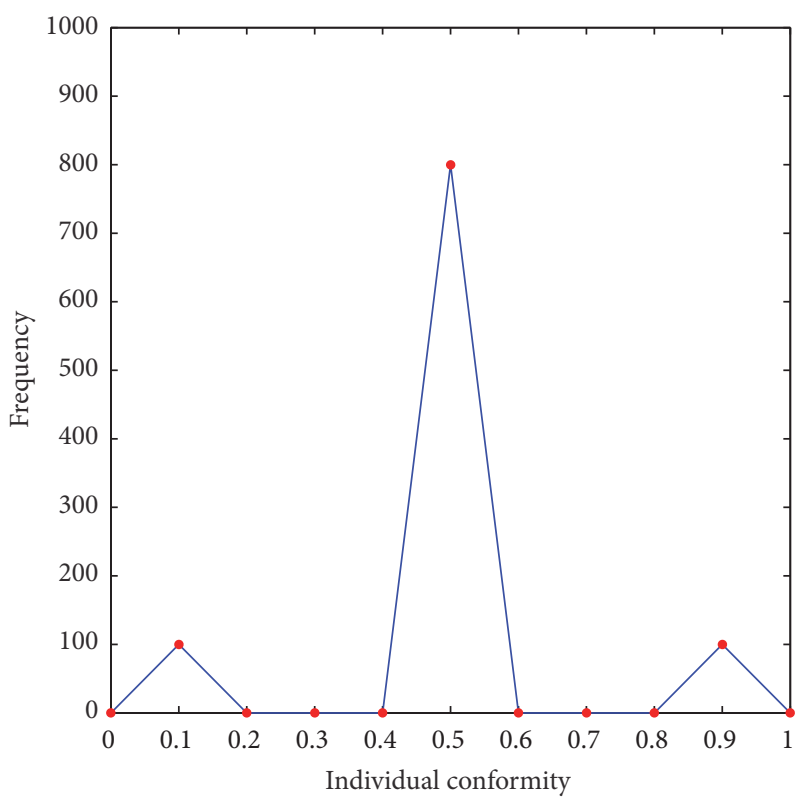

FIGURE 1: Individual conformity distribution.

$\left(X_{i}, Y_{j}\right)$ is assigned to agent $i$ to denote the geographic position of agent $i$. The maximum value of both $X_{i}$ and $Y_{i}$ is $d_{\max }$. The physical distance between agent $i$ and agent $j$ is quantified as

$$
d(i, j)=\sqrt{\left(X_{i}-X_{j}\right)^{2}+\left(Y_{i}-Y_{j}\right)^{2}} \text {. }
$$

An interactive radius parameter $-\delta$ is assigned to agent $i$ on behalf of the communication ability. $\delta$ determines the reference collection one has access to.

(3) Public Authority. Generally speaking, individuals of high prestige or great social power would be more widespread and have greater acceptance among common people. To represent the diversity of attributes among individuals, a public authority parameter is assigned to each agent regarding social influence on neighbors in opinion exchange, which is related to the individual attributes.

To quantify the individual public authority [16], we refer to the fifth population census data of China and consider age, economic level, and educational attainment as the three most important factors in determining public authority. Other issues are considered as a random factor. The public authority of the individual $i$ is computed as

$$
\mathrm{PA}_{i}=\left[w_{1}, w_{2}, w_{3}, w_{4}\right] \cdot\left[S_{\text {age }}, S_{\text {economy }}, S_{\text {edu }}, S_{\text {other }}\right]^{T},
$$

where $w_{1}+w_{2}+w_{3}+w_{4}=1$ and $S_{\text {age }}, S_{\text {economy, }} S_{\text {edu }}, S_{\text {other }}$, respectively, denote the age, economic level, educational attainment, and a random factor. The values of public authority are a real number between $[0,1]$, distributed as Figure 2. A public authority is entirely different from social power [13]. The definition of social power is $\operatorname{SP}_{i}=\left(k_{i}\right)^{\alpha}$, where $k$ is the degree of the individual $i$. Social power only considers the heterogeneity impact of network topology, without taking

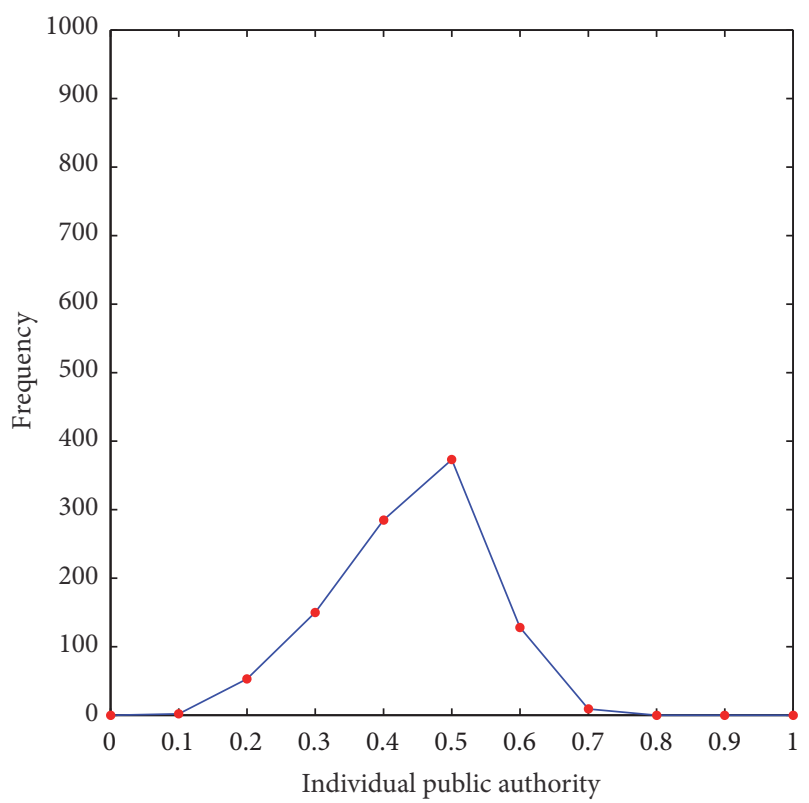

FIGURE 2: Individual public authority distribution.

into account individual attributes. Public authority proposed in our model is only related to individual attributes.

2.2. Model Assumptions. Before we model the evolution process, we give a description of some assumptions about the model.

(a) Opinion evolution follows the principle of bounded confidence, which means that only people with similar opinions talk to each other.

(b) As individual resources are finite; it is impossible for any individual to get in touch with all the other persons and there is a limit on the communication space, assumed as spatial constraints.

(c) Different social individuals manifest various social attributes and social power.

(d) Public media is ignored during the process of opinion evolution.

2.3. Algorithm Description. By the above hypothesis, we now present the HI model according to the behavior characteristics of individual interaction. Consider a system consisting of $N$ agents. Assume that agent $i$ keeps a real number representing his/her initial opinion $x_{i}(t)$ randomly distributed in the range of $0 \sim 1$, where 0 stands for extreme agreement and 1 stands for extreme disagreement. All of the agents have the same confidences radius $\mathcal{E}$ and the same interactive radius $\sigma$. At each discrete time $t$, opinion update process is performed once including the reference collection calculation, exchange collection calculation, and opinion update in turn.

(1) Reference Collection Calculation. Under the condition of spatial constraints, only individuals within a certain range of space would be considered when the individual $i$ interacts 
in perspective. Moreover, the collection constituted by these individuals is called a reference collection of the individual $i$. For a given time $t$, the physical distance radius $d_{i}$ of the individual $i$ defines the size of the reference collection. The reference collection Distance $(i)$ of individual $i$ can be calculated as follows:

$$
\text { Distance }(i):\{j \mid 0<d(i, j)<\delta, 1 \leq j \leq N, j \neq i\} \text {. }
$$

In the above equation, the physical distance radius $\delta$ is a fixed constant.

(2) Exchange Collection Calculation. According to the size of their trust in the neighborhood, an individual with a certain scope of opinion difference would be chosen. The collection constituted by these individuals is called exchange collection of the individual $i$. For a given time $t$, the exchange collection Neighbor( $i$ ) of node $i$ is decided by the radius of trust $\varepsilon_{i}$ and reference collection Distance $(i)$. Its can be calculated as follows:

$$
\begin{aligned}
& \text { Neighbor }(i) \text { : } \\
& \qquad\left\{j|| x_{i}(t)-x_{j}(t) \mid<\varepsilon_{i}, j \in \operatorname{Distance}(i), j \neq i\right\} .
\end{aligned}
$$

As can be seen from the equation, the individual is excluded from the exchange collection by the filtration of reference collection in HI model. However, the individual himself must be included in the exchange collection of classical limited trust model. Therefore, the exchange collection of HI model may be empty.

(3) Opinion Update. When the exchange collection is determined, the system begins to exchange opinions. In the next moment, the individual $i$ maintains his current opinion with probability $1-\lambda_{i}$ and is affected by his trusted friend with probability $\lambda_{i}$; the influence of individual $j$ on individual $i$ is proportional to the public authority $\mathrm{PA}_{j}$ of the individual $j$. When the exchange collection of individual $i$ is empty, the opinion of the individual $i$ remains unchanged. The opinion of individual $i$ at $t+1$ time is expressed as

$$
\begin{aligned}
& x_{i}(t+1) \\
& = \begin{cases}\left(1-\lambda_{i}\right) x_{i}(t)+\lambda_{i} \sum_{j \in N_{i}} \frac{\mathrm{PA}_{j}}{\sum_{j} \mathrm{PA}_{j}} \times x_{j}(t), & \text { Neighbor }(i) \neq \phi \\
x_{i}(t), & \text { Neighbor }(i)=\phi .\end{cases}
\end{aligned}
$$

Opinion updating formula shows that the new opinion of the individual in the next moment is formed under the joint action of the individual's current opinion, his personality characteristics, the opinion, and public authority of trusted friends.

An algorithm flowchart of our proposed model is shown in Figure 3. Our model operates as follows.

Step 1 (parameter initialization). Set appropriate parameters including the number of individuals, physical distance radius, confidence radius, and evolution time.

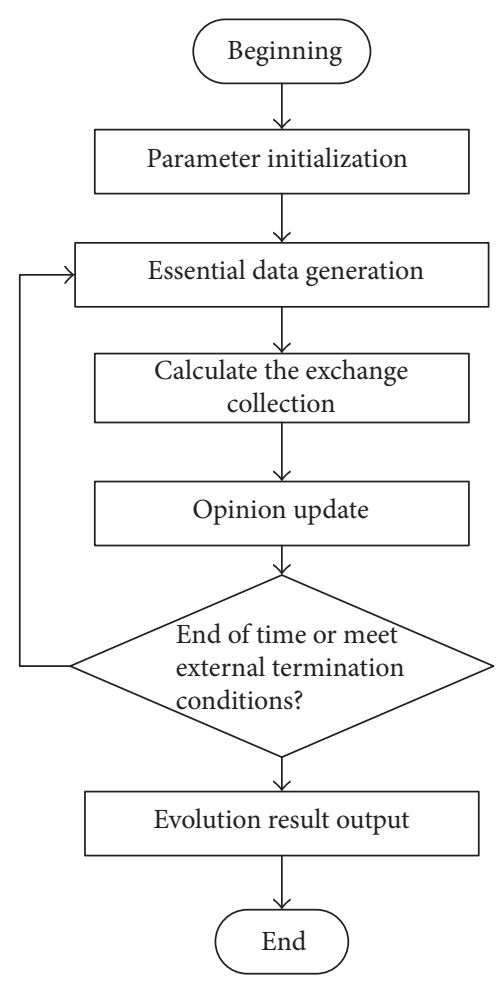

FIGURE 3: HI model algorithm flowchart.

Step 2 (essential data generation). According to statistical proportion, essential data is generated to initialize the basic attributes of each agent.

Step 3. Calculate the exchange collection and the current agent change opinion with according to (3) and (4).

Step 4 (opinion update is completed). If the exchange collection is not empty, then the current individual updates his opinion according to (5) at next step or his opinion will remain unchanged. Loop Steps 3 and 4 until all of the opinions achieve stable states. Then the evolution is ended.

Step 5 (evolution result output). Output the time evolution result of the individual opinions, initial opinion distribution curve, and terminal opinion distribution curve.

\section{The Opinion Research of HI Model in Full-Connected Network and Scale-Free Network}

According to the fifth population census data of China [17], this paper utilizes the statistical data of age proportion, population proportion in different geographical positions, and education level proportion, respectively, in juvenile, youth, middle age, and old age. We obtained the economic condition in the various regions. Geographical distribution proportion, age proportion, and education proportion are presented in Table 1. 
TABLE 1: The statistical data of the fifth population consensus.

\begin{tabular}{|c|c|c|c|c|c|c|c|c|}
\hline \multicolumn{9}{|c|}{ Geographical distribution proportion (\%) } \\
\hline East & South & West & North & Middle & Southeast & Northeast & Southwest & Northwest \\
\hline 22.86 & 11.04 & 0.23 & 11.77 & 20.46 & 2.74 & 8.42 & 20.95 & 1.53 \\
\hline \multicolumn{2}{|c|}{ Gender (\%) } & \multicolumn{7}{|c|}{ Age $(\%)$} \\
\hline Male & Female & Infants & Child & Juvenile & \multicolumn{2}{|c|}{ Youth } & Middle-aged & Elderly \\
\hline 51.63 & 48.37 & 3.17 & 5.08 & 14.67 & \multicolumn{2}{|c|}{37.61} & 29.76 & 9.71 \\
\hline \multicolumn{5}{|c|}{ Proportion of education among the juvenile (\%) } & \multicolumn{4}{|c|}{ Proportion of education among the youth (\%) } \\
\hline Illiteracy & Primary & Intermediate & Senior & Illiteracy & \multicolumn{2}{|c|}{ Primary } & Intermediate & Senior \\
\hline 2.68 & 75.79 & 21.53 & 0 & 2.04 & \multicolumn{2}{|c|}{21.63} & 70.35 & 5.98 \\
\hline \multicolumn{5}{|c|}{ Proportion of education among the middle-aged (\%) } & \multicolumn{4}{|c|}{ Proportion of education among the elderly (\%) } \\
\hline Illiteracy & Primary & Intermediate & Senior & Illiteracy & & & Intermediate & Senior \\
\hline 8.88 & 38.26 & 49 & 3.86 & 47.55 & & & 13.58 & 2.05 \\
\hline
\end{tabular}

The simulation environment is designed as follows:

(1) Essential data of 1000 nodal attributes is generated according to the statistical proportion of the fifth national census.

(2) The platform for essential data generation is developed in C\#. The simulation environment is Matlab $2010 \mathrm{~b}$ and the database is SQLServer 2008.

(3) According to the fifth census data, we can obtain the individual's physical location $(d \max =1000)$. The physical distance between agent $i$ and agent $j$ is calculated from formula (4). The physical distance distribution is shown in Figure 4.

3.1. The Comparison of HI Model and KH Model. To compare the differences between HI model and classical KH model, this paper compares the opinion evolution of the two models in all connected networks and scale-free networks, under the same individual attributes (confidence radius, physical distance, and initial opinion) distribution. Selections of confidence radius are $0.05,0.2$, and 0.3 . The initial opinion is random distribution between $(0,1)$. Individual authority and conformity are according to the distribution of Figures 1 and 2, respectively. As shown in Figure 4, 80\% of the physical distance between two individuals is more than 600 . Therefore, the interactive radius is set at 600 .

The evolution result in the full-connected network is shown in Figure 5. The Figure indicates that the opinion evolution will be from discrete to polarization, finally reaching a consensus in both $\mathrm{HI}$ model and $\mathrm{KH}$ model, with the increase of confidence radius. The evolutions of public opinion of two models are similar, and the stable opinions are the same. However, the convergence time is very different. The convergence time of $\mathrm{HI}$ model is longer than the convergence time of $\mathrm{KH}$ mode. As the limitation of the interactive radius in HI model, the evolution process tends to be slow.

The evolution result in scale-free networks is shown in Figure 6. The opinion evolution will be from discrete to polarization, finally reaching a consensus in both HI model and $\mathrm{KH}$ model, with the increase of confidence radius. However, in a scale-free network, no matter what kind of

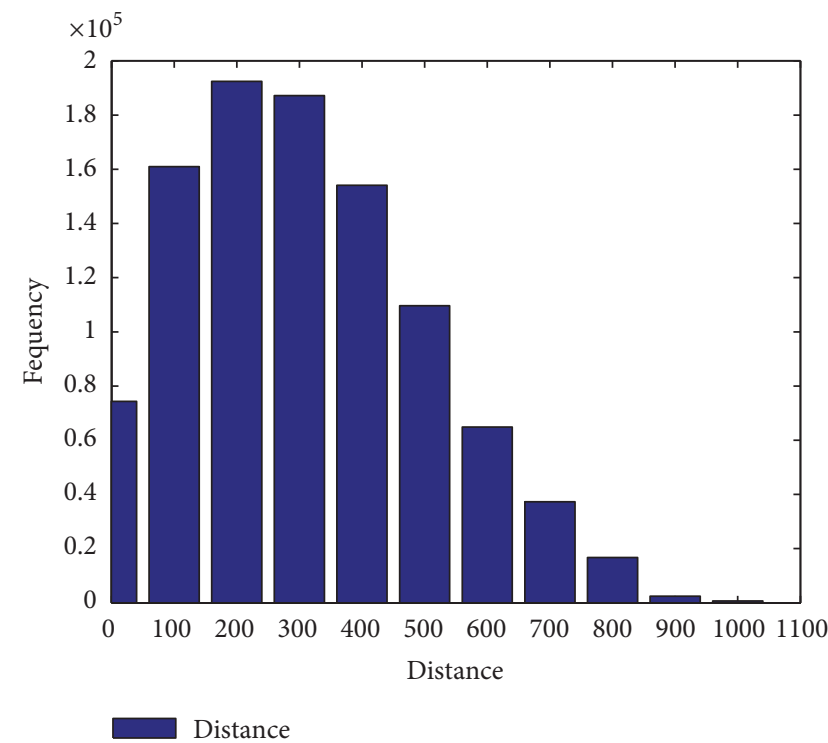

FIgure 4: The physical distance distribution.

model, the convergence time is longer than that in the fullconnected network in the same situation. This is because the number of individual joint nodes is reduced, and it cannot reach a steady state in a short time, which leads to an increase in the convergence time.

From the above experimental result, it shows that the evolution time will be longer when interactive radius limits the $\mathrm{HI}$ model. In $\mathrm{KH}$ model, without the limitation of interactive radius, individuals can have more easy access to all other individual opinions in the system and update their opinion in a relatively short time. The global opinion could reach a steady state quickly. Also, $\mathrm{KH}$ model ignores the interpersonal influence and individual cognitive differences and just puts the average value of the neighbor's opinion which is less than a confidence threshold with an own opinion as for the next moment opinion. However, the influence of different neighbors on him is not the same, when individual takes part in opinion interaction process in the real world. It makes the individual exchange opinion under 

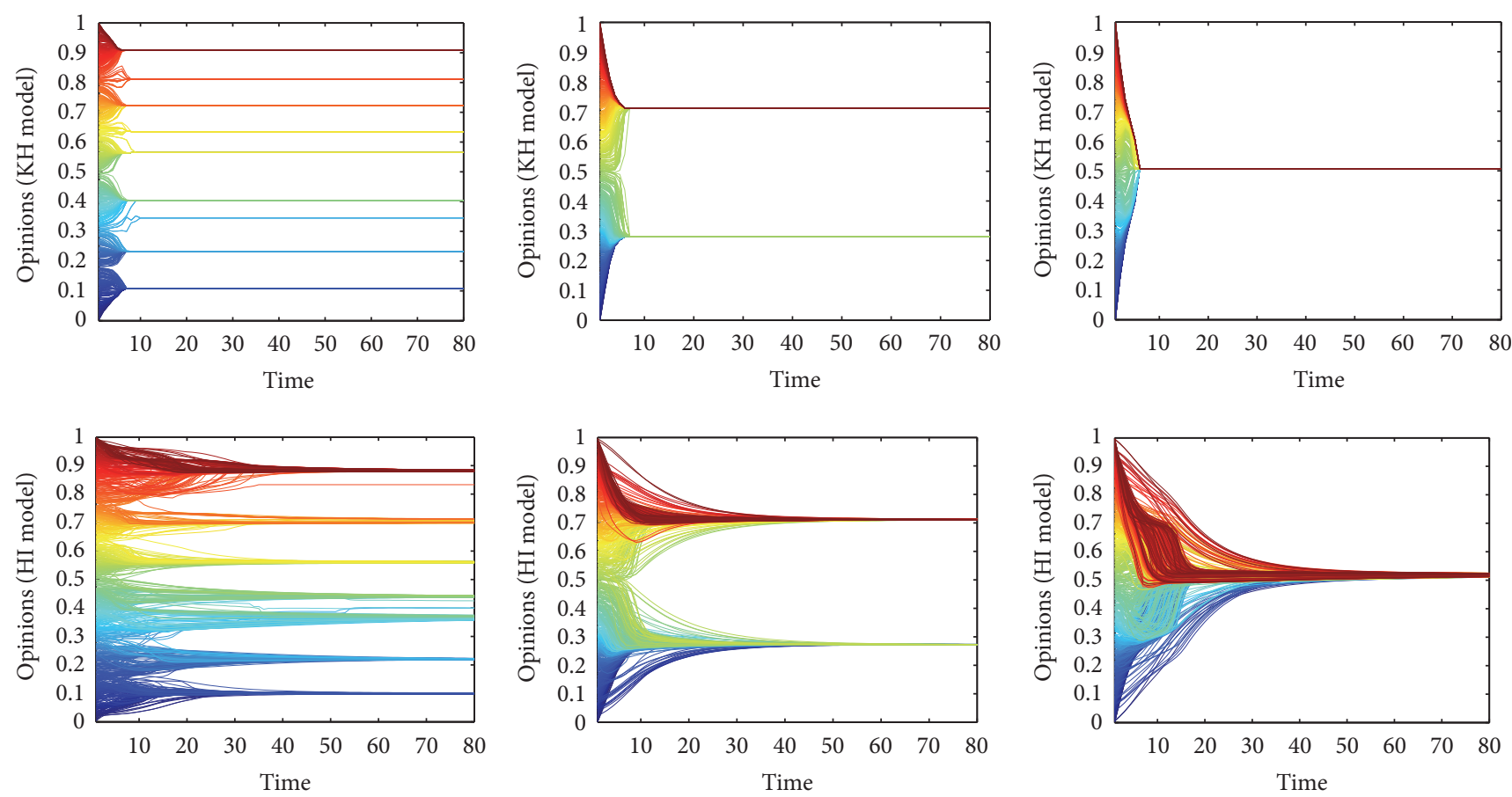

(a) $\varepsilon=0.05$

(b) $\varepsilon=0.2$

(c) $\varepsilon=0.3$

FIGURE 5: The comparison of public opinion evolution between $\mathrm{KH}$ model (the above three figures) and HI model (the three figures below) in full-connected network. $\varepsilon$ : (a) $\varepsilon=0.05$, (b) $\varepsilon=0.2$, and (c) $\varepsilon=0.3$.
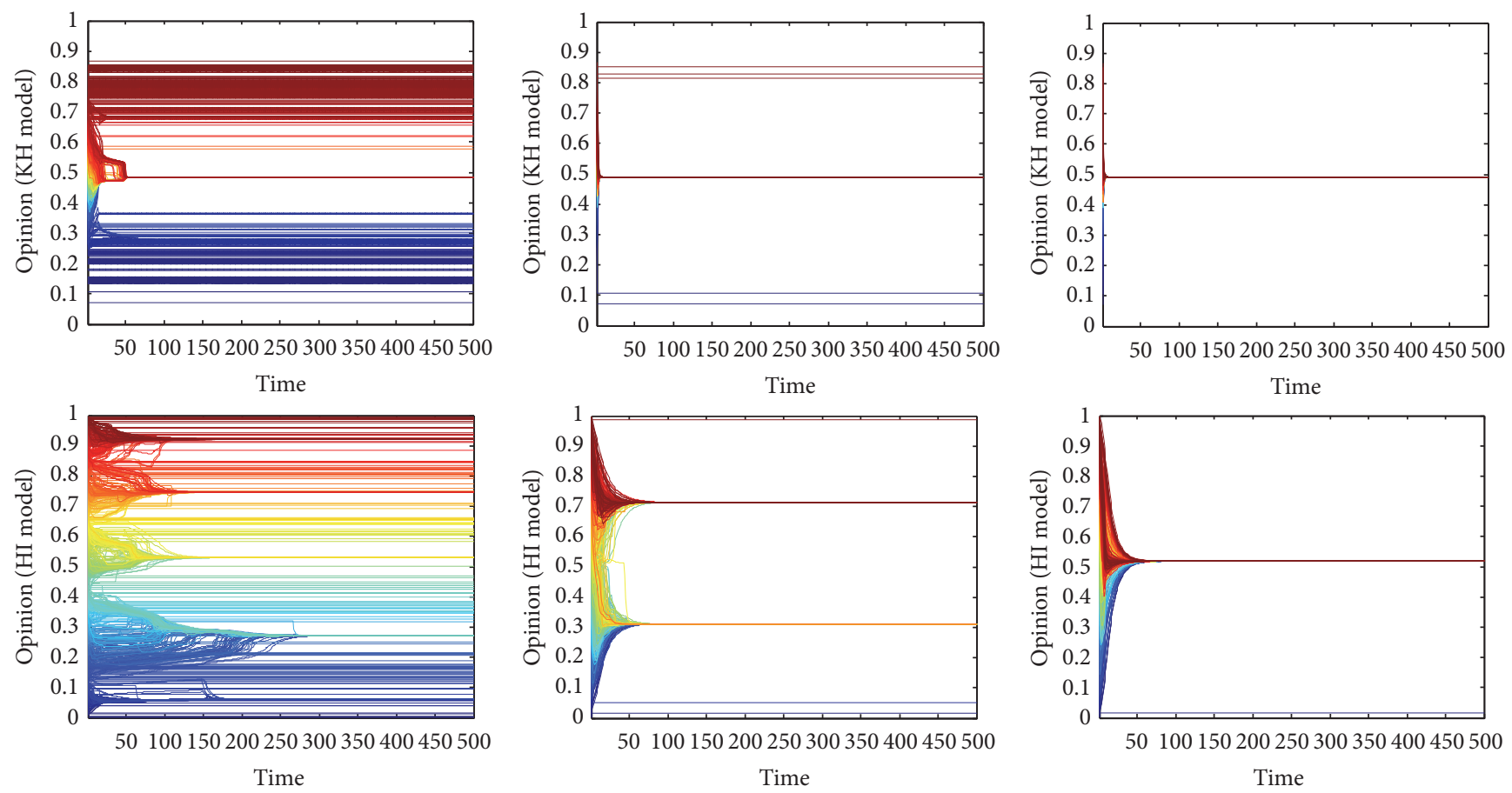

(a) $\varepsilon=0.05$

(b) $\varepsilon=0.2$

(c) $\varepsilon=0.3$

FIGURE 6: The comparison of public opinion evolution between KH model (the above three figures) and HI model (the three figures below) in the scale-free network. $\varepsilon$ : (a) $\varepsilon=0.05$, (b) $\varepsilon=0.2$, and (c) $\varepsilon=0.3$. 

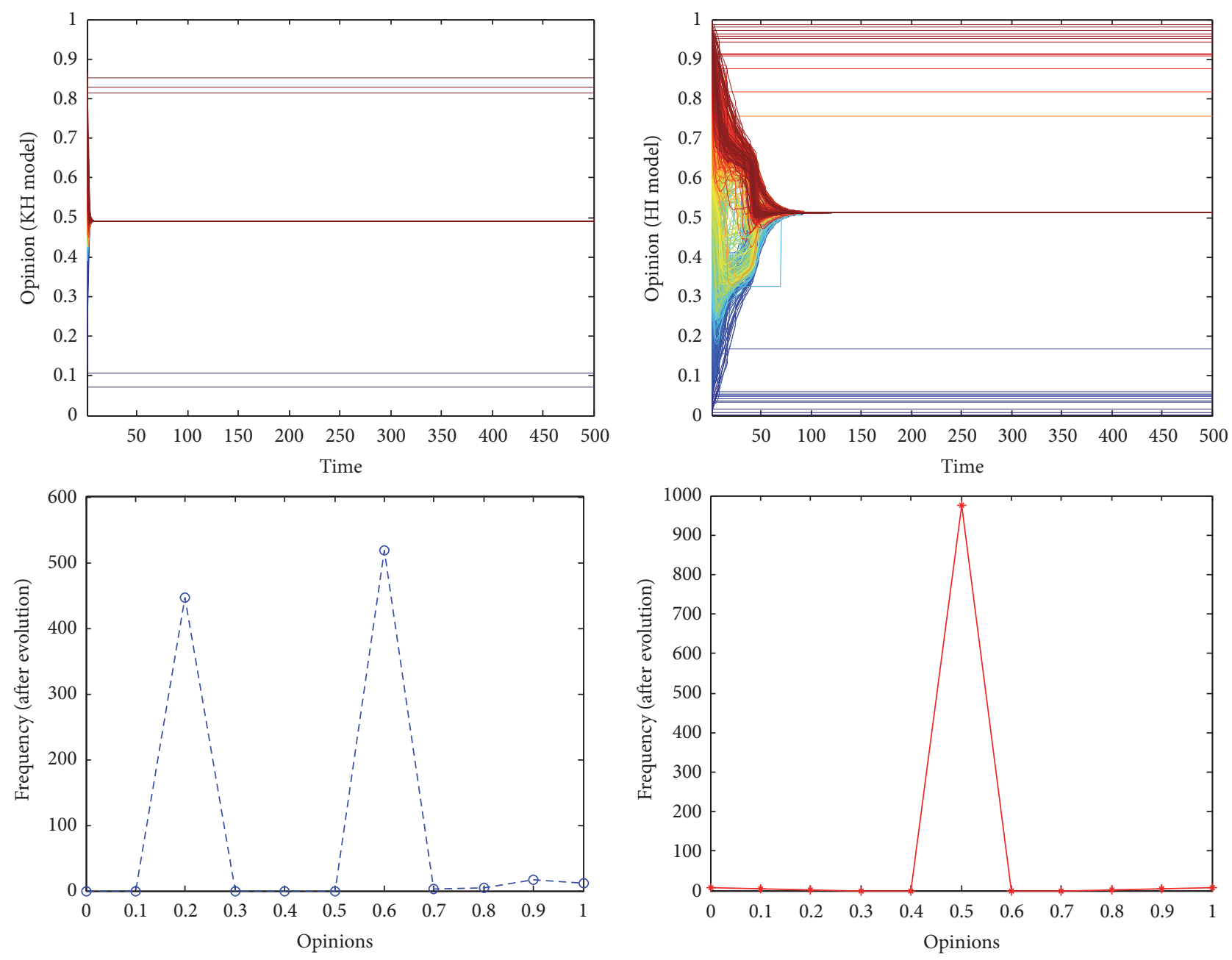

$-\ominus-\mathrm{KH}$ model

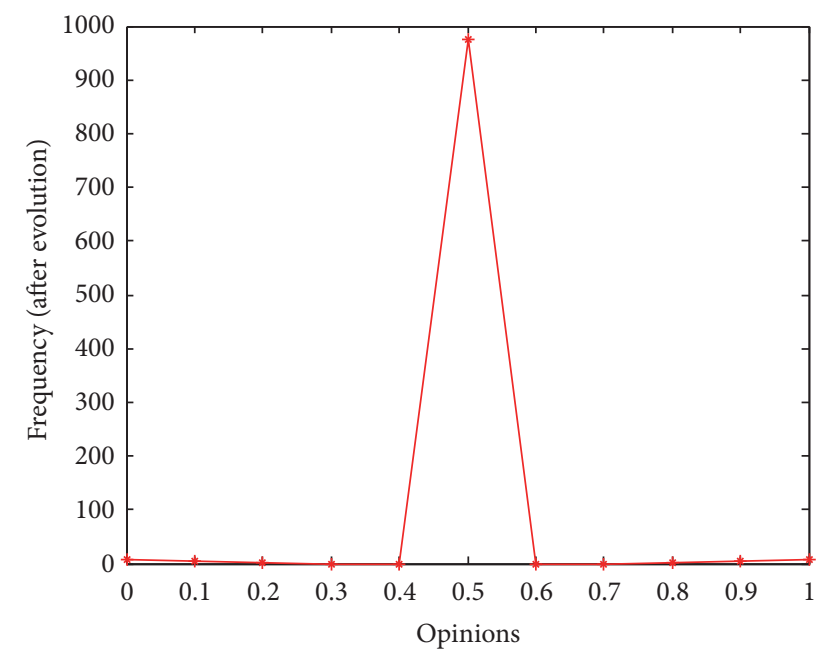

$\rightarrow$ HI model

(a) $\mathrm{KH}$ model

(b) HI model

FIGURE 7: The opinion evolution in scale-free network. (a) KH model and (b) HI model. $\varepsilon=0.2, \delta=400$.

limited communication range, increasing the difficulty of the individual to identify the overall environment of public opinion. It causes difficulty in forming their final opinion, and public opinion convergence takes a long time. Based on the actual situation, agents using the improved model can use interactive choice agents to update their opinion under the limitation of the individual's heterogeneity and cognition degree, although forming the consensus or a stable state needs a long time. Eventually, evolution effect, such as discrete, polarization, and consensus, is similar to classical $\mathrm{KH}$ model.

\subsection{The Different Interactive Radius Experiments in HI Model.} In real life, the different physical distance will affect the interaction between the individuals. This paper considers evolution under a different range of interactive radius between $\mathrm{HI}$ model and classical $\mathrm{KH}$ model. Considering the scalefree network edge which is similar to individuals in real life, the simulation experiments are conducted in the scale-free networks. Because the opposition between two views (such as vote) in real life, namely, the polarization phenomenon, is common, the confidence radius of two models will be set to 0.2 , which is easy to present the phenomenon. The initial opinion distributions of two models are taking the same random distribution.

Figure 7 shows the difference between the opinion evolution of HI model (interactive radius $\delta=400$ ) and $\mathrm{KH}$ model.

The figure shows that the convergence time of HI model is slower than the convergence time of $\mathrm{KH}$ model and the final cluster number increase. The main reason is due to the limitation of confidence radius $\varepsilon$ and interactive radius $\delta$ which make the interactive agent number decrease in HI model. To describe the largest cluster in the steady state of public opinion evolution, Yang et al. [18] defined $S$ index as the ratio of the individual number of the largest cluster and overall cluster, Luo et al. [19] defined $G$ index, and Chen et al. [11] defined $C$ as a final number of controlled agents. We choose $C$ to describe the individual number of the largest cluster in the steady state. Figure 8 shows, under different interactive radius, the individual number of the largest cluster $(C)$ in two models. 

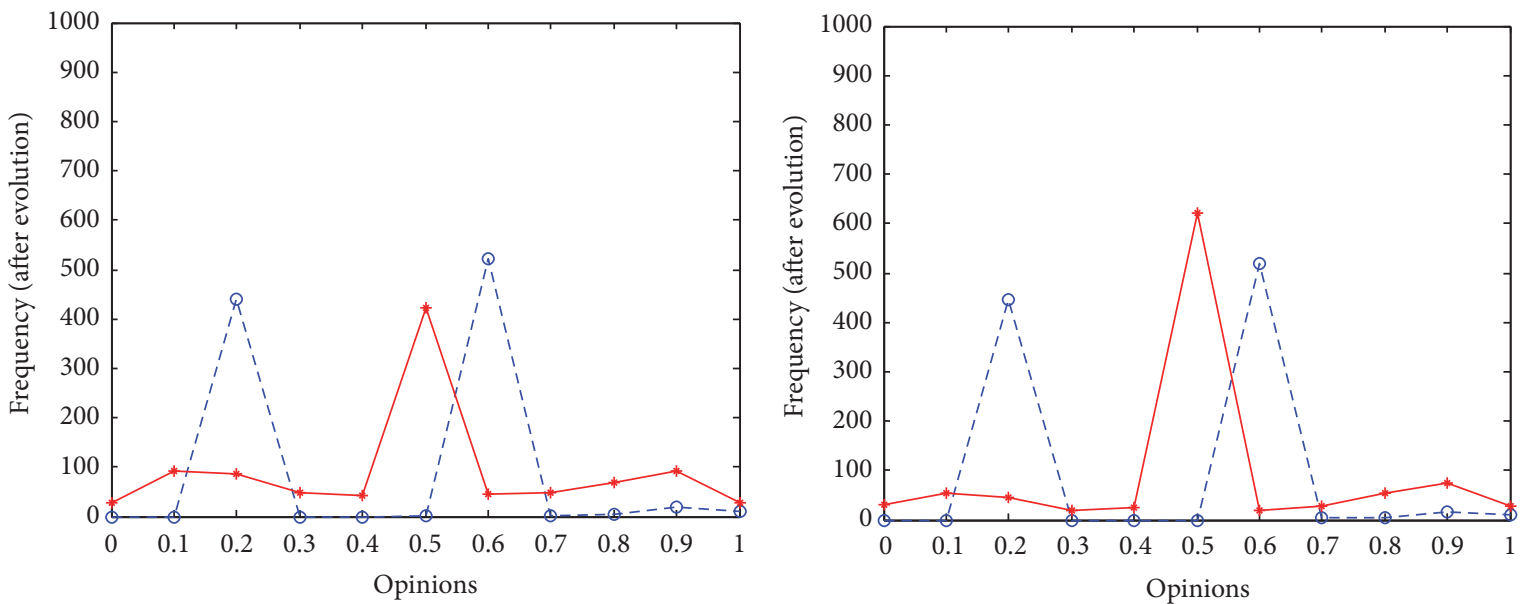

$-\ominus-$ KH model

*- HI model

$-\ominus-\mathrm{KH}$ model

$\rightarrow$ HI model

(a) $\delta=120$
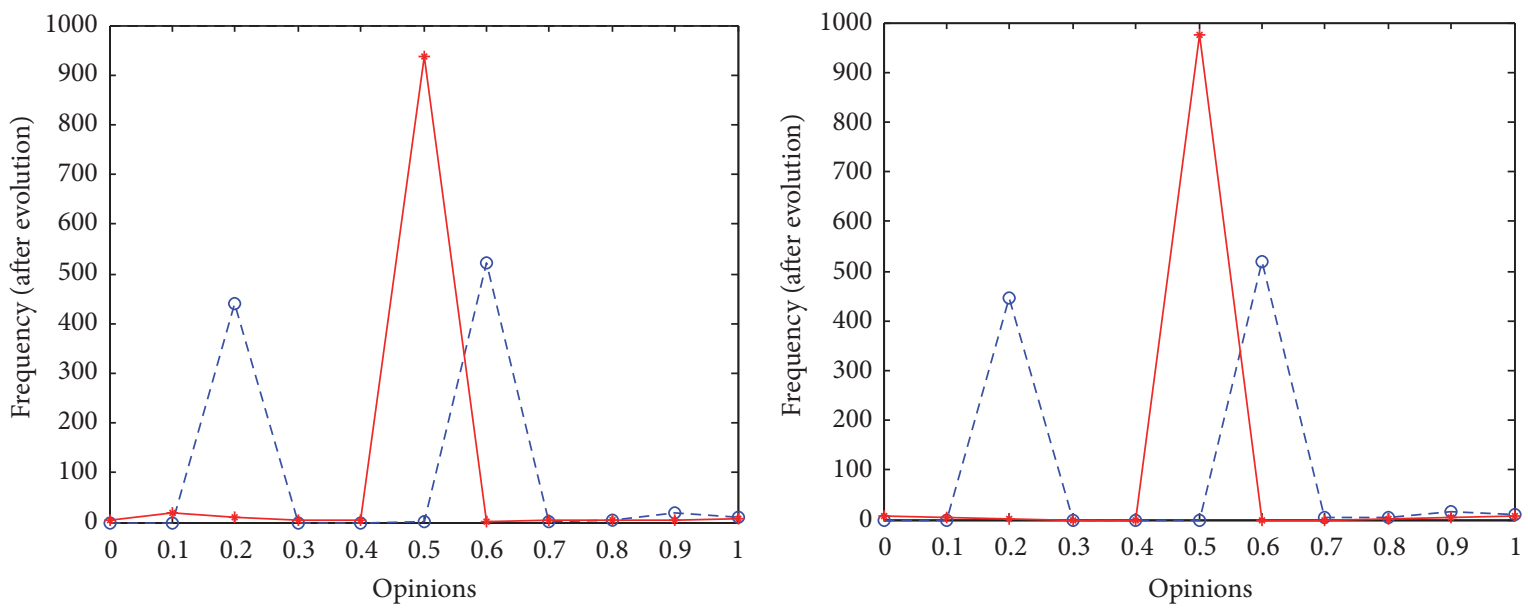

- $\ominus-$ KH model

- $\ominus-$ KH model

$\rightarrow$ HI model

(c) $\delta=300$

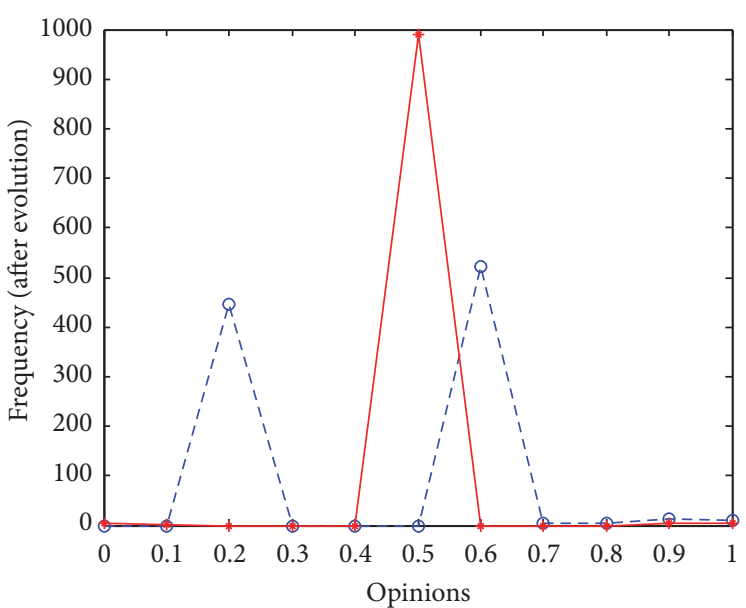

- $\ominus-\mathrm{KH}$ model

$\rightarrow$ HI model

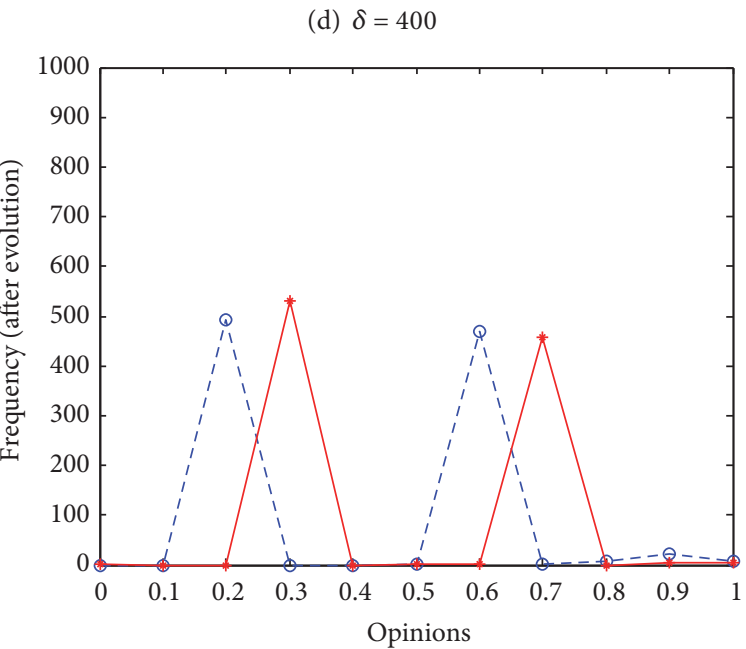

- $\ominus-\mathrm{KH}$ model

* $\mathrm{HI}$ mode

(e) $\delta=520$

(f) $\delta=550$

FIGURE 8: The final opinion distribution of HI model and KH model in scale-free network, $\varepsilon=0.2$. (a) $\delta=120$, (b) $\delta=150$, (c) $\delta=300$, (d) $\delta$ $=400$, (e) $\delta=520$, and (f) $\delta=550$. 
Figure 8 shows that when the interactive radius is between 150 and 520 , the individual number of the largest cluster $(C)$ of $\mathrm{HI}$ model is greater than the individual number of the largest cluster $(C)$ of $\mathrm{KH}$ model. Figure 8(a) shows that the individual number of the largest cluster $(C)$ of $\mathrm{HI}$ model is clearly less than the individual number of the largest cluster $(C)$ of $\mathrm{KH}$ model after interactive rate becomes less than 150 . Figure 8(f) shows that after the interactive radius becomes greater than 520 , the HI model will eventually show the tendency of the polarization, and the individual number of the largest cluster $(C)$ is not better than the individual number of the largest cluster $(C)$ of $\mathrm{KH}$ model. This means that the interactive radius of HI model can influence the individual number of the largest cluster $(C)$ of public opinion. In this case, because of the limitation of interactive radius, most of the nodes can be in the direction of the consensus when confidence radius is small.

From the above, when confidence radius is small, most agents will have a tendency to reach consensus in HI model, but most agents appear to have the trend of opinions polarization in $\mathrm{KH}$ model. This is due to the fact that the public opinion evolution of $\mathrm{KH}$ model is only determined by the confidence radius, without considering the limitation of actual physical distance in the society. In HI model, both interactive radius and confidence radius affect the agent's interactive range. When the two parameters are limited to a proper range, most agents will be a consensus by local communication because of the mutual influence.

\section{Conclusion}

Based on the classic $\mathrm{KH}$ model, this paper proposes a new evolution model: heterogeneous interaction model (HI model), in the limitation of physical distance, individual conformity, and authority. The HI model conducts an opinion evolution in the full-connected network and the scale-free network, and the result is compared with the KH model. The result of experiment reveals that the evolutional efficiency of the network model will become slow after adding the limitation of the interactive radius, but the final evolutional situation of $\mathrm{HI}$ model and $\mathrm{KH}$ model is analogous. In addition, when other conditions are consistent and interactive radius is in a certain range, most agents of $\mathrm{HI}$ model will be consensus, but the opinions of agents will be polarized in the $\mathrm{KH}$ model. It means that we can limit the interactive radius to make most agents in consensus and control the final guidance of public opinion.

Based on the above experiments, the results prove that the individual heterogeneity and interactive radius have a significant influence in the public opinion evolution, and under the certain constraints of the condition, it can control the individual number of the largest cluster $(C)$ of the evolution of public opinion. Most of the previous researches ignore the individual heterogeneity and the physical distance between individuals and only consider the confidence radius, so it makes public opinion evolution model too simple, and the evolution process is different with the real-world situations. HI model takes the individual heterogeneity and interactive distance into account, and the evolution process conforms to real-world data wherein the evolution time increases and the final point cluster increases. This means that the $\mathrm{HI}$ model is more reasonable in real life in describing the evolution of public opinion and can more effectively observe and predict the actual circumstances of the public opinion evolution by the HI model. In real life, the final result of public opinion evolution is that winner is who have more supporters, namely, the individual number of the largest cluster $(C)$ is more than $50 \%$ of the whole, and HI model can effectively control the individual number of the largest cluster $(C)$ by controlling the largest interactive distance. To some extent, the public opinion evolution of HI model reveals that the distance between the individuals has a significant influence on public opinion in consensus in real life. This also means that in real life, the authority can change the scope of interactive radius between the individuals to control the final trend of public opinion.

After that, we will conduct a more in-depth research in the individual heterogeneity of the HI model.

\section{Conflicts of Interest}

The authors declare that there are no conflicts of interest regarding the publication of this article.

\section{Acknowledgments}

This work was supported by the National Natural Science Foundation of China under Grant nos. 71571081, 61540032, and 91324203.

\section{References}

[1] R. Xiao and T. Yu, "A multi-agent simulation approach to rumor spread in virtual commnunity based on social network," Intelligent Automation and Soft Computing, vol. 17, no. 7, pp. 859-869, 2011.

[2] R. Xiao, Y. Zhang, and Z. Huang, "Emergent computation of complex systems: A comprehensive review," International Journal of Bio-Inspired Computation, vol. 7, no. 2, pp. 75-97, 2015.

[3] P. Novoa-Hernández, C. C. Corona, and D. A. Pelta, "Self-adaptation in dynamic environments-A survey and open issues," International Journal of Bio-Inspired Computation, vol. 8, no. 1, pp. 1-13, 2016.

[4] G. Deffuant, D. Neau, F. Amblard, and G. Weisbuch, "Mixing beliefs among interacting agents," Advances in Complex Systems, vol. 3, no. 1-4, pp. 87-98, 2000.

[5] R. Hegselmann and U. Krause, "Opinion dynamics and bounded confidence: models, analysis and simulation," Journal of Artificial Societies \& Social Simulation, vol. 5, no. 3, article 2, 2002.

[6] K. Sznajd-Weron and J. Sznajd, "Opinion evolution in closed community," International Journal of Modern Physics C, vol. 11, no. 6, pp. 1157-1165, 2000.

[7] S. Fortunato and D. Stauffer, "Computer simulations of opinions and their reactions to extreme events," in Extreme Events in Nature and Society, pp. 233-257, Springer, Berlin, Germany, 2006. 
[8] D. Stauffer, "Sociophysics simulations II: opinion dynamics," AIP Conference Proceedings, vol. 779, no. 1, pp. 56-68, 2005.

[9] G.-R. Chen, W.-D. Cai, H.-J. Xu, P.-X. Yan, and J.-P. Wang, "High-effect priority bounded confidence model for network opinion evolution," Journal of Shanghai Jiaotong University, vol. 47, no. 1, pp. 155-160, 2013.

[10] J. Lorenz, "Heterogeneous bounds of confidence: meet, discuss and find consensus!", Complexity, vol. 15, no. 4, pp. 43-52, 2010.

[11] X. Chen, X. Xiong, M. Zhang, and W. Li, "Public authority control strategy for opinion evolution in social networks," Chaos, vol. 26, no. 8, Article ID 083105, 2016.

[12] H. Liang, Y. Yang, and X. Wang, "Opinion dynamics in networks with heterogeneous confidence and influence," Physica A: Statistical Mechanics and Its Applications, vol. 392, no. 9, pp. 2248-2256, 2013.

[13] M. Jalili, "Social power and opinion formation in complex networks," Physica A: Statistical Mechanics and Its Applications, vol. 392, no. 4, pp. 959-966, 2013.

[14] S. Li and S. Zhang, "Leader and follower: agents in an opinion dynamics and bounded confidence model on the stochastic movement world," in Proceedings of the 2nd International Conference on Computational Intelligence and Natural Computing (CINC '10), pp. 50-54, September 2010.

[15] M. A. Javarone, "Social influences in opinion dynamics: the role of conformity," Physica A: Statistical Mechanics and Its Applications, vol. 414, pp. 19-30, 2014.

[16] X. Chen, L. Zhang, and W. Li, "A network evolution model for chinese traditional acquaintance networks," IEEE Intelligent Systems, vol. 29, no. 5, pp. 5-13, 2014.

[17] "National Bureau of Statistics of the People's Republic of China[EB/OL]," http://www.stats.gov.cn/tjsj/pcsj/.

[18] W. Yang, L. Cao, X. Wang, and X. Li, "Consensus in a heterogeneous influence network," Physical Review E-Statistical, Nonlinear, and Soft Matter Physics, vol. 74, no. 3, Article ID 037101, 2006.

[19] S. Luo, Y. Du, P. Liu, Z. Xuan, and Y. Wang, "A study on coevolutionary dynamics of knowledge diffusion and social network structure," Expert Systems with Applications, vol. 42, no. 7, pp. 3619-3633, 2015. 


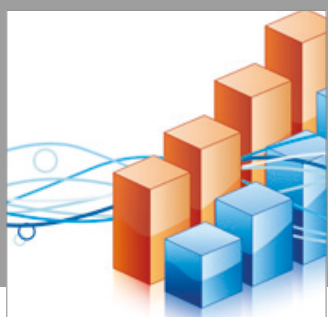

Advances in

Operations Research

vatersals

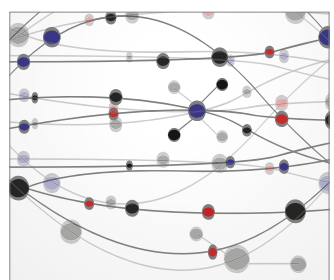

\section{The Scientific} World Journal
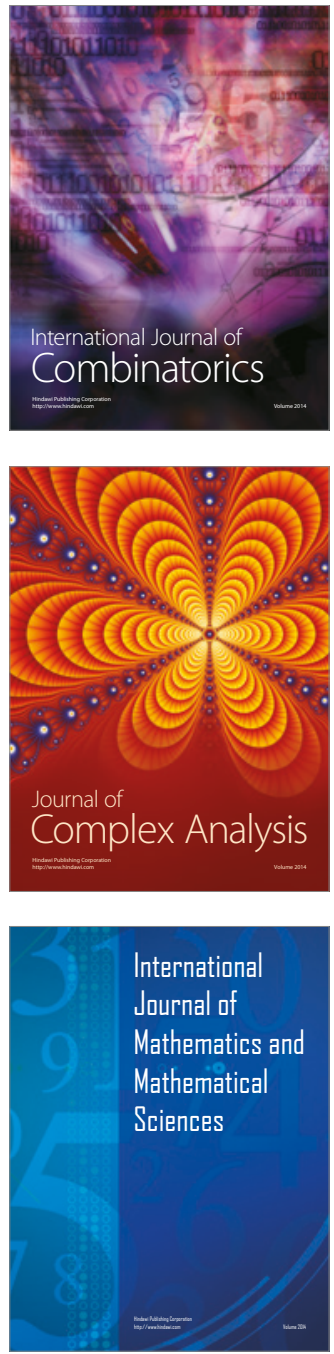
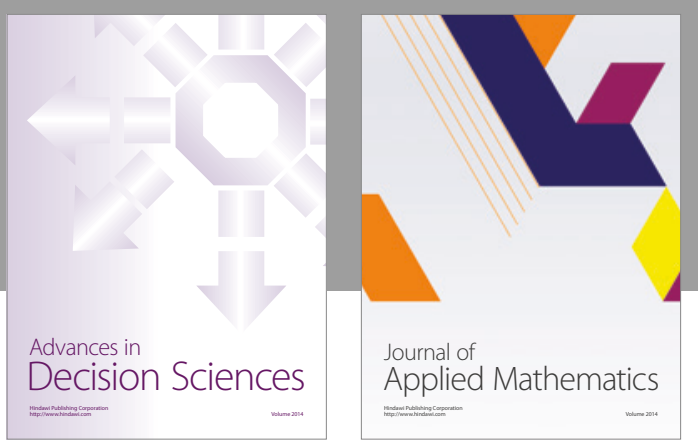

Algebra

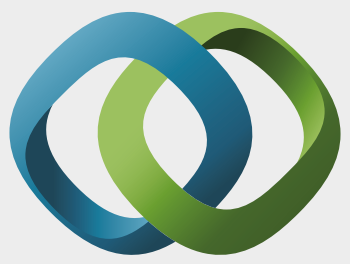

\section{Hindawi}

Submit your manuscripts at

https://www.hindawi.com
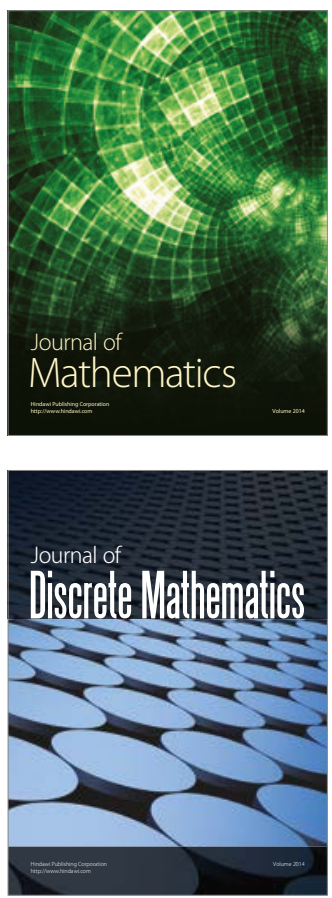

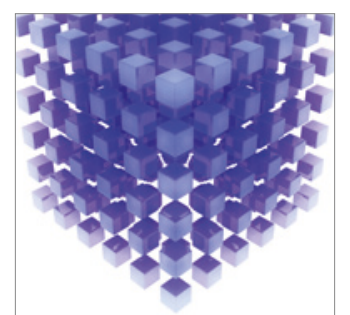

Mathematical Problems in Engineering
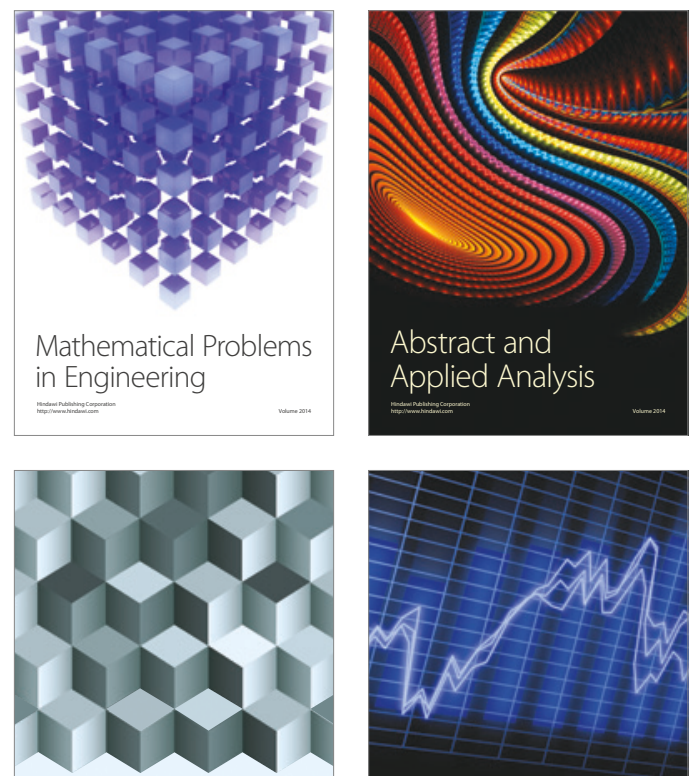

Journal of

Function Spaces

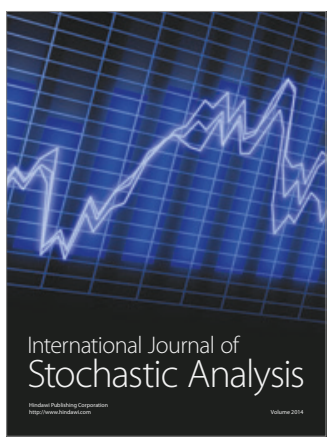

Probability and Statistics
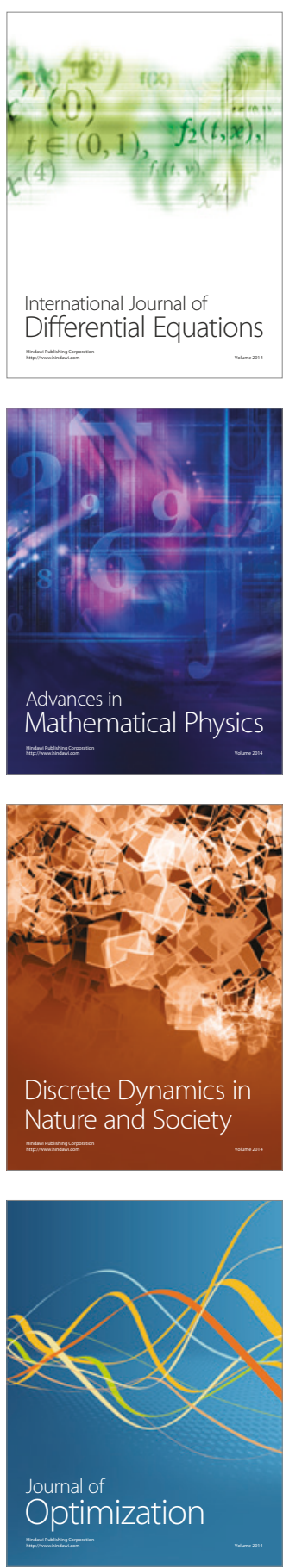